\title{
Adverse impact of depression and anxiety on mortality in patients with breast cancer
}

\author{
Seon-Young Kim ${ }^{1,2}$, Min Jhon ${ }^{2}$, David W. Kissane ${ }^{3,4}$ \\ ${ }^{1}$ Department of Psychiatry, Chonnam National University Medical School, Gwangju, Republic of Korea; ${ }^{2}$ Mental Health Clinic, Chonnam National \\ University Hwasun Hospital, Hwasun, Republic of Korea; ${ }^{3}$ Department of Psychiatry, School of Clinical Sciences at Monash Health, Monash \\ University, Clayton, Victoria, Australia; ${ }^{4}$ School of Medicine, University of Notre Dame Australia and Cunningham Centre, St Vincent's Sydney, \\ NSW, Australia \\ Correspondence to: Seon-Young Kim. Department of Psychiatry, Chonnam National University Medical School, Gwangju, Republic of Korea. \\ Email: sykimpsy@chonnam.ac.kr. \\ Provenance and Peer Review: This article was commissioned by the editorial office, Translational Cancer Research. The article did not undergo external \\ peer review. \\ Comment on: Shim EJ, Lee JW, Cho J, et al. Association of depression and anxiety disorder with the risk of mortality in breast cancer: A National \\ Health Insurance Service study in Korea. Breast Cancer Res Treat 2020;179:491-8.
}

Submitted May 29, 2020. Accepted for publication Jun 09, 2020.

doi: $10.21037 /$ tcr-20-2205

View this article at: http://dx.doi.org/10.21037/tcr-20-2205

Depression and anxiety are common in patients with cancer. As in the reports regarding the association between depression and mortality in various physical conditions (1), there have been similar investigations of these issues in patients with cancer (2). However, these investigations have yielded mixed results regarding mortality, likely due to methodological differences among the studies. For example, the timing of diagnosis of psychiatric disease, i.e., before versus after the cancer diagnosis can affect the results (3). For the choice of assessment measures, the evidence for an association between depression and mortality was weaker in studies that used structured interviews than in those that used dimensional scales (1). Adjustment for confounding variables can also affect the results. The well-known prognostic factors in breast cancer are primary tumor size, presence of regional lymph nodes, distant metastases, histologic grade, and hormone receptor status. Other important confounding variables include social inequality, obesity, cigarette smoking, alcohol intake and exercise. These factors are not reliably documented in retrospective studies, unless drawn from large linkage studies such as those conducted by the Danish Cancer Society (4).

In a previous meta-analysis of 25 independent studies, the mortality rate was up to $39 \%$ above average in patients diagnosed with major or minor depression [unadjusted relative risk (RR), 1.39; 95\% confidence interval (CI), 1.10-1.89] (5). Another recent meta-analysis by Wang and colleagues (2) of 51 cohort studies reported significant impacts of depression and/or anxiety on cancer-specific mortality (adjusted RR, 1.21; 95\% CI, 1.16-1.26) and allcause mortality in patients with various types of cancer (adjusted RR, 1.24; 95\% CI, 1.13-1.35). However, in subgroup analysis of seven studies of breast cancer, depression and anxiety were not significantly associated with mortality.

Several biobehavioural pathways have been suggested to be possible links between psychological distress and tumour progression. Non-adherence to cancer treatment, including endocrine therapy, and negative lifestyle factors, such as smoking, physical inactivity and alcohol consumption, can impact cancer progression indirectly $(4,6,7)$. Depression may impact the immune system of patients due to impaired activity of natural killer cells and cell-mediated immunity. Dysregulation of hypothalamic-pituitary-adrenal axis activity and activation of the automatic nervous system could increase inflammation and oxidative/nitrosative stress (6).

Given the concerns regarding the association between depression and mortality in patients with cancer, the question of whether treatment of depression could improve survival has been raised. There have been several 
randomised controlled trials regarding the effects on mortality of psychosocial interventions in patients with comorbid cancer and depression, as a primary or secondary outcome, but the results were inconsistent; therefore, there is still debate concerning this issue $(8,9)$. Meanwhile, there have been few investigations regarding the potential benefit of antidepressant treatment in survival in patients with cancer. Most studies used a retrospective design, which makes it difficult to identify causal relationships.

In breast cancer, there have been reports of an increased risk of mortality, rather than decreased mortality, associated with antidepressant use. Various types of antidepressants, e.g., selective serotonin reuptake inhibitors (SSRIs) and tricyclic antidepressants (TCAs), have been reported to increase breast cancer mortality in women, although conflicting results have been reported (10). Among the SSRIs, paroxetine has attracted attention with regard to increasing mortality in patients with breast cancer due to its possible interaction with tamoxifen via inhibition of the cytochrome P450 2D6 isoenzyme, its alteration of oestrogen responsiveness, or its oestrogenic effect (10). Although this has not yet been proven, it is prudent to exert caution when considering paroxetine and related SSRIs using the P450 2D6 metabolic pathway for patients with breast cancer.

\section{Time of first-onset of mental illness in relation to cancer diagnosis}

Shim et al. used National Health Insurance Service (NHIS) data from South Korea to examine the associations of depression, anxiety and antidepressant treatment with mortality in patients with breast cancer (11). The prevalence rates of depression and anxiety were lower than in previous studies performed in Western countries, and also much lower than in another nationwide South Korean study that used NHIS data (12). This could have been due to the exclusion of cases of depression or anxiety diagnosed 1 year before the breast cancer diagnosis. It is important to be specific regarding the timing of the depression/anxiety diagnosis in relation to that of cancer when investigating the impact of these comorbidities on mortality. The effects of mental disorders with first onset after cancer diagnosis may differ from those of recurrent mental disorders, with the former probably being a product of cancer-specific stress (3). In a nationwide cohort study, mental disorders with first onset after cancer diagnosis were shown to increase cancerspecific mortality, while recurrent mental disorders had no such effect (3).

\section{Association of depressive and anxiety disorders with mortality in patients with breast cancer}

Regarding patients with breast cancer, there have been some reports specifically related to the effects of depression and/or anxiety on the mortality rate (Table 1) (11,13-21). Among ten studies, three analyzed cancer-specific mortality and the others analyzed all-cause mortality. The study by Shim et al. also did not analyse cancer-specific mortality. When analyzing mortality, it is recommended to investigate cancer-specific mortality to identify the direct impact of depression on cancer outcomes, because depression is associated with a higher mortality rate in the general population (5). Even though the association of depression with cancer-specific mortality was not found to be significant, the association with all-cause mortality might be significant if non-cancer-related mortality had a sufficiently large effect size (22).

Including the study by Shim et al., five studies investigated the impact of depression and anxiety on mortality $(11,14,17,19,21)$. Only the study by Shim et al. analyzed the specific impacts of depression and anxiety and the impact of their co-occurrence. Depression and anxiety were independently associated with mortality in patients with breast cancer, and their co-occurrence further increased the risk (11).

As mentioned above, adjusting recognised confounding factors is also important. The results of the study by shim et al. were adjusted by sex, age, place of residence, income level, comorbid illness, type of breast cancer, disability, and receipt of chemotherapy/radiation therapy/hormonal therapy/target therapy (11). However, the results were not fully adjusted for several important confounding factors to analyze mortality. For example, the severity of cancer at first diagnosis is a risk factor for mortality that affects results. In a nationwide retrospective cohort study conducted in Denmark, preoperative depression was associated with elevated mortality in late-stage breast cancer, while postoperative depression was associated with increased mortality in early stage breast cancer (15). The study by Shim et al., investigated the cancer type, i.e., cancer $v s$. carcinoma-in-situ, but did not differentiate between early $v s$. late breast cancer. Furthermore, significantly more carcinoma-in-situ cases were included in the nondepression/non-anxiety group than in the other groups. Although this difference was adjusted for by covariates, whether advanced cancer cases were similarly distributed between the groups remains unknown. Furthermore, other 


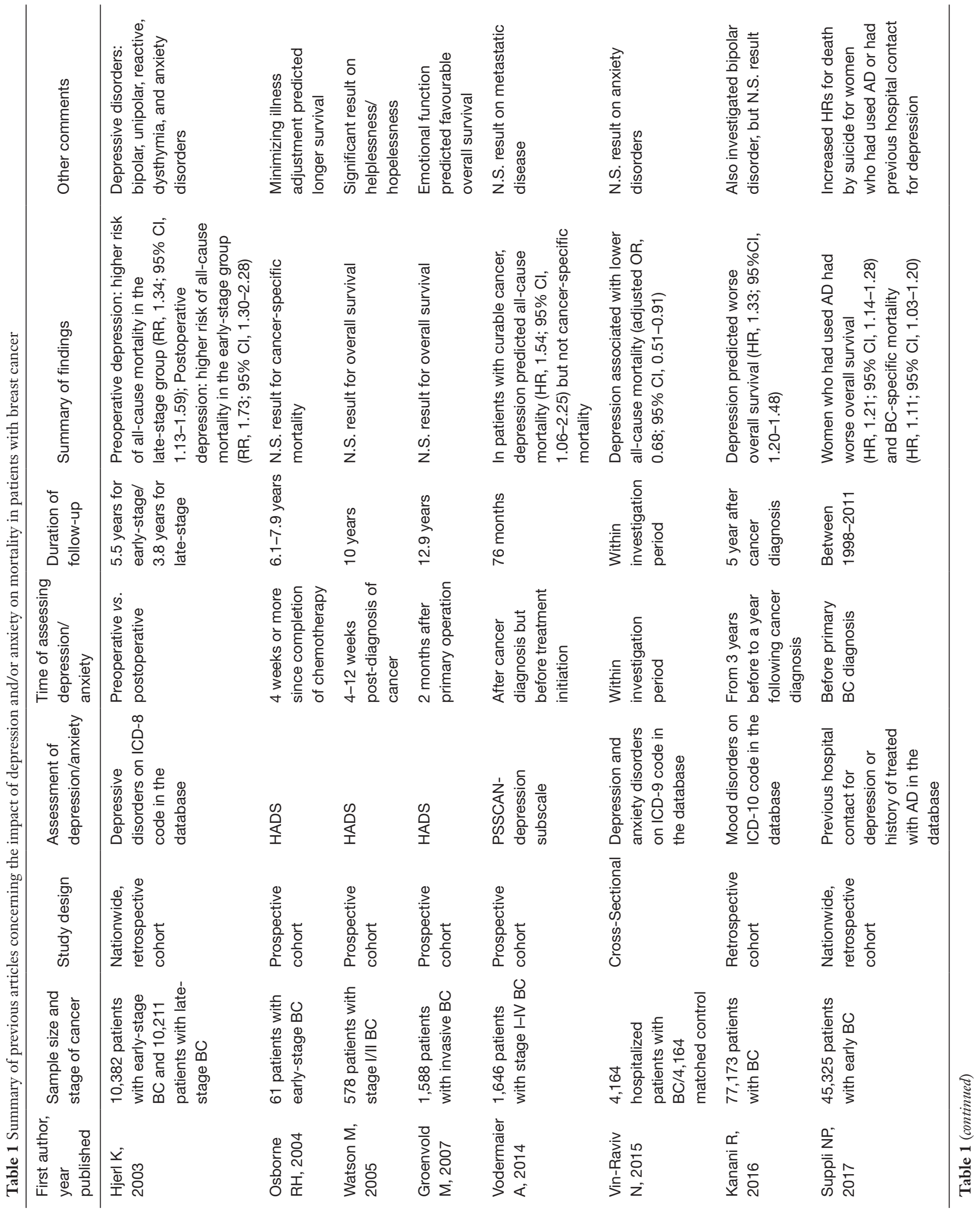




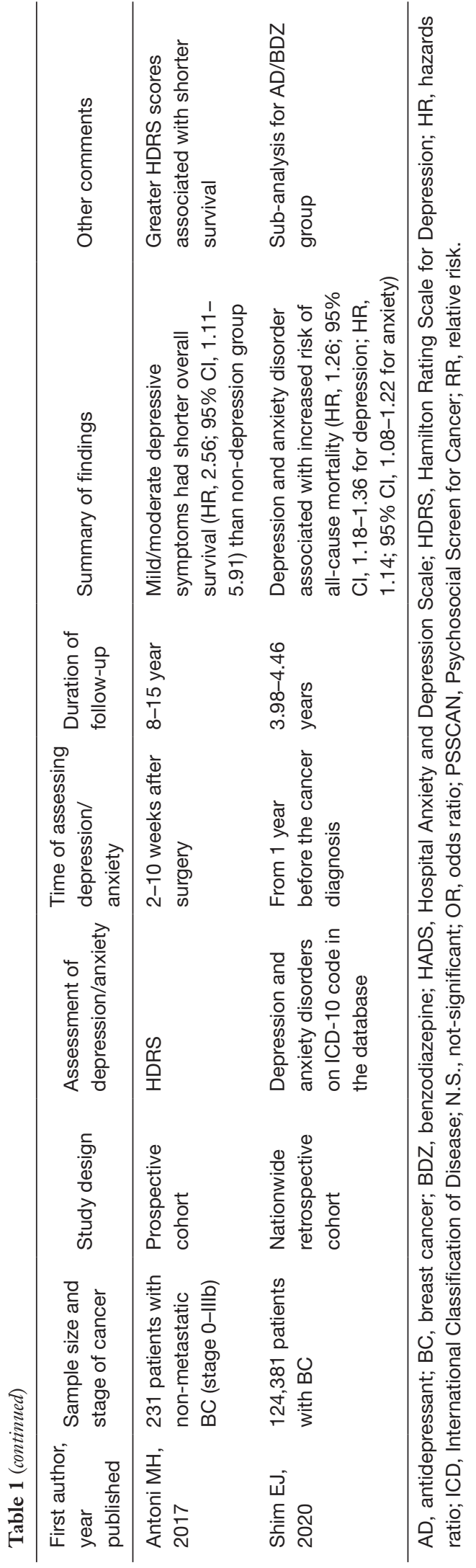

factors such as involvement of axillary lymph nodes, distant metastasis, and other socio-behavioral confounding factors were not investigated.

\section{Impact of antidepressants on mortality in patients with breast cancer}

Shim et al. reported that antidepressant treatment was related to reduced cancer mortality. This finding was unique and noteworthy, but caution is required in its interpretation. The study did not specifically focus on the impact of the treatment of depression or anxiety, but rather on the association of the use of psychiatric medication with mortality in patients with breast cancer. A few studies have investigated the associations between antidepressants and mortality in patients with cancer; the results varied between increased and decreased mortality $(23,24)$. But regardless of the controversial results found in previous studies, it is necessary to pay attention to the application of "antidepressant treatment" used in the study of Shim and colleagues. The expression "antidepressant treatment" included not only "antidepressants" but also "anxiolytics", such as benzodiazepines in the study. This may answer the question raised by Chen et al. in a letter regarding why "antidepressants treatment" were administered to patients without formal depressive and anxiety disorders in that study (25): anxiolytics are widely recommended to control various symptoms other than anxiety or depression in patients with cancer. It ought not to be concluded that those antidepressants or benzodiazepines actually decreased the risk of mortality in patients with breast cancer because the mortality rate was increased in the patients receiving antidepressant treatment among a non-depressed and nonanxious group in the study of Shim et al. However, Shim et al. reported "antidepressant treatment" may attenuate the risk because the mortality rate was lower in the patients receiving antidepressant treatment among the group with the depression and anxiety, compared to one with the same diagnosis but not receiving antidepressant treatment. While this is quite encouraging, the paper did not provide the full results of pairwise comparisons, i.e., pairwise over strata, on the log-rank test to compare more than two groups. Only the results of a log-rank test comparing these groups with a reference group (i.e., a group without anxiety or depression that received no antidepressant treatment) were provided.

In a similar previous nationwide study, higher adherence to antidepressants was associated with decreased all-cause mortality in patients with various type of cancer (24). 
However, among patients with breast cancer, the results differed from those of another population-based study which showed that patients with long-term SSRI use had higher mortality than their non-SSRI-using counterparts, regardless of any prior diagnosis of depression (23). Given the limitations inherent in the retrospective and/ or observational designs of these studies, randomised controlled trials with mortality as the primary outcome are required for more definitive conclusions.

\section{Conclusion and future directions}

Determining the impact of mental illness on mortality in patients with breast cancer is very important. It is also important to determine whether appropriate mental health interventions could reduce mortality in these patients, but previous studies reported conflicting results and had methodological limitations. Nationwide studies using health insurance databases have the benefit of large sample sizes, but it is difficult to determine causal relationships and control variables in a retrospective design. However, the results could provide a basis for future prospective studies or randomised controlled trials. To investigate the impact on mortality, the timing of the depression/anxiety diagnosis relative to the cancer diagnosis, the cancer-specific mortality, and full adjustment for confounding variables are recommended.

\section{Acknowledgments}

Funding: None.

\section{Footnote}

Conflicts of Interest: All authors have completed the ICMJE uniform disclosure form (available at http://dx.doi. org/10.21037/tcr-20-2205). The authors have no conflicts of interest to declare.

Ethical Statement: The authors are accountable for all aspects of the work in ensuring that questions related to the accuracy or integrity of any part of the work are appropriately investigated and resolved.

Open Access Statement: This is an Open Access article distributed in accordance with the Creative Commons Attribution-NonCommercial-NoDerivs 4.0 International License (CC BY-NC-ND 4.0), which permits the non- commercial replication and distribution of the article with the strict proviso that no changes or edits are made and the original work is properly cited (including links to both the formal publication through the relevant DOI and the license). See: https://creativecommons.org/licenses/by-nc-nd/4.0/.

\section{References}

1. Machado MO, Veronese N, Sanches M, et al. The association of depression and all-cause and cause-specific mortality: an umbrella review of systematic reviews and meta-analyses. BMC Med 2018;16:112.

2. Wang YH, Li JQ, Shi JF, et al. Depression and anxiety in relation to cancer incidence and mortality: a systematic review and meta-analysis of cohort studies. Mol Psychiatry 2020;25:1487-99.

3. Zhu J, Fang F, Sjölander A, et al. First-onset mental disorders after cancer diagnosis and cancer-specific mortality: a nationwide cohort study. Ann Oncol 2017;28:1964-9.

4. Larsen SB, Kroman N, Ibfelt EH, et al. Influence of metabolic indicators, smoking, alcohol and socioeconomic position on mortality after breast cancer. Acta Oncol 2015;54:780-8.

5. Satin JR, Linden W, Phillips MJ. Depression as a predictor of disease progression and mortality in cancer patients: a meta-analysis. Cancer 2009;115:5349-61.

6. Bortolato B, Hyphantis TN, Valpione S, et al. Depression in cancer: The many biobehavioral pathways driving tumor progression. Cancer Treat Rev 2017;52:58-70.

7. Haskins CB, McDowell BD, Carnahan RM, et al. Impact of preexisting mental illness on breast cancer endocrine therapy adherence. Breast Cancer Res Treat 2019;174:197-208.

8. Kissane DW. Letting go of the hope that psychotherapy prolongs cancer survival. J Clin Oncol 2007;25:5689-90.

9. Mirosevic S, Jo B, Kraemer HC, et al. "Not just another meta-analysis": Sources of heterogeneity in psychosocial treatment effect on cancer survival. Cancer Med 2019;8:363-73.

10. Nevels RM, Gontkovsky ST, Williams BE. ParoxetineThe Antidepressant from Hell? Probably Not, But Caution Required. Psychopharmacol Bull 2016;46:77-104.

11. Shim EJ, Lee JW, Cho J, et al. Association of depression and anxiety disorder with the risk of mortality in breast cancer: A National Health Insurance Service study in Korea. Breast Cancer Res Treat 2020;179:491-8.

12. Heo J, Chun M, Oh YT, et al. Psychiatric comorbidities 
among breast cancer survivors in South Korea: a nationwide population-based study. Breast Cancer Res Treat 2017;162:151-8.

13. Antoni MH, Jacobs JM, Bouchard LC, et al. Post-surgical depressive symptoms and long-term survival in nonmetastatic breast cancer patients at 11-year follow-up. Gen Hosp Psychiatry 2017;44:16-21.

14. Groenvold M, Petersen MA, Idler E, et al. Psychological distress and fatigue predicted recurrence and survival in primary breast cancer patients. Breast Cancer Res Treat 2007;105:209-19.

15. Hjerl K, Andersen EW, Keiding N, et al. Depression as a prognostic factor for breast cancer mortality. Psychosomatics 2003;44:24-30.

16. Kanani R, Davies EA, Hanchett N, et al. The association of mood disorders with breast cancer survival: an investigation of linked cancer registration and hospital admission data for South East England. Psychooncology 2016;25:19-27.

17. Osborne RH, Sali A, Aaronson NK, et al. Immune function and adjustment style: do they predict survival in breast cancer? Psychooncology 2004;13:199-210.

18. Suppli NP, Johansen C, Kessing LV, et al. Survival After Early-Stage Breast Cancer of Women Previously Treated for Depression: A Nationwide Danish Cohort Study. J Clin Oncol 2017;35:334-42.

19. Vin-Raviv N, Akinyemiju TF, Galea S, et al. Depression

Cite this article as: Kim SY, Jhon M, Kissane DW. Adverse impact of depression and anxiety on mortality in patients with breast cancer. Transl Cancer Res 2020;9(7):4046-4051. doi: $10.21037 /$ tcr-20-2205 and Anxiety Disorders among Hospitalized Women with Breast Cancer. PLoS One 2015;10:e129169.

20. Vodermaier A, Linden W, Rnic K, et al. Prospective associations of depression with survival: a populationbased cohort study in patients with newly diagnosed breast cancer. Breast Cancer Res Treat 2014;143:373-84.

21. Watson M, Homewood J, Haviland J, et al. Influence of psychological response on breast cancer survival: 10-year follow-up of a population-based cohort. Eur J Cancer 2005;41:1710-4.

22. Ko A, Kim K, Sik Son J, et al. Association of pre-existing depression with all-cause, cancer-related, and noncancerrelated mortality among 5-year cancer survivors: a population-based cohort study. Sci Rep 2019;9:18334.

23. Busby J, Mills K, Zhang SD, et al. Selective serotonin reuptake inhibitor use and breast cancer survival: a population-based cohort study. Breast Cancer Res 2018;20:4.

24. Shoval G, Balicer RD, Feldman B, et al. Adherence to antidepressant medications is associated with reduced premature mortality in patients with cancer: A nationwide cohort study. Depress Anxiety 2019;36:921-9.

25. Chen HL, Du L, Li XM. Re: Association of depression and anxiety disorder with the risk of mortality in breast cancer. Breast Cancer Res Treat 2020;179:767-8. 